Ann. Zootech., I980, 29 (1), I3-2I.

\title{
Etude du rationnement de la poule pondeuse avec distribution de régimes à teneurs différentes en protéïnes
}

\author{
B. LECLERCQ et J. C. BLUM \\ avec la collaboration technique de Solange GuII,Avmin, \\ Gérard GuY et Serge NevorT \\ Station de Rechcrches Avicoles \\ Centre de Recherches de Tours, I.N.R.A., \\ Nouzilly, 37380 Monnaie (France)
}

\section{Résumé}

Au cours de 2 expériences portant chacune sur 540 pondeuses de type Rhode Island Red, on a distribué à volonté ou en quantité restreinte des régimes à teneurs différentes en protéines. Le rationnement ne modifie pas ou augmente même un peu le poids moyen de l'œuf. Il améliore l'indice de consommation, mais conduit toujours à une diminution du nombre d'œufs pondus quel que soit le tatix protidique.

Pendant les premiers mois d'application du rationnement, les effets observés dépendent seulement de la quantité de protéines consommées; le besoin est estimé alors à $20 \mathrm{~g} / \mathrm{j}$ et par poule (maïs, soja, méthionine). Cependant, pour l'ensemble de la ponte, le besoin de la poule ne dépasse pas $18 \mathrm{~g}$ et les résultats ne peuvent être entièrement expliqués en invoquant une déficience azotée variable. Il demeure un effet spécifique du rationnement.

\section{Introduction}

Le rationnement des poules de type Rhode-Wyandotte pendant la ponte permet une économie d'aliment. Il améliore l'efficacité alimentaire et réduit l'engraissement. Malheureusement, il tend à diminuer légèrement la production d'œufs (Bougon et MÉvel, I972; LECLERCQ, Bouchardeau et BLUM, I975). Nous avons voulu savoir si le rationnement intervenait seulement comme une privation de constituants énergétiques et dans quelle mesure le besoin en protéines demeurait constant. 


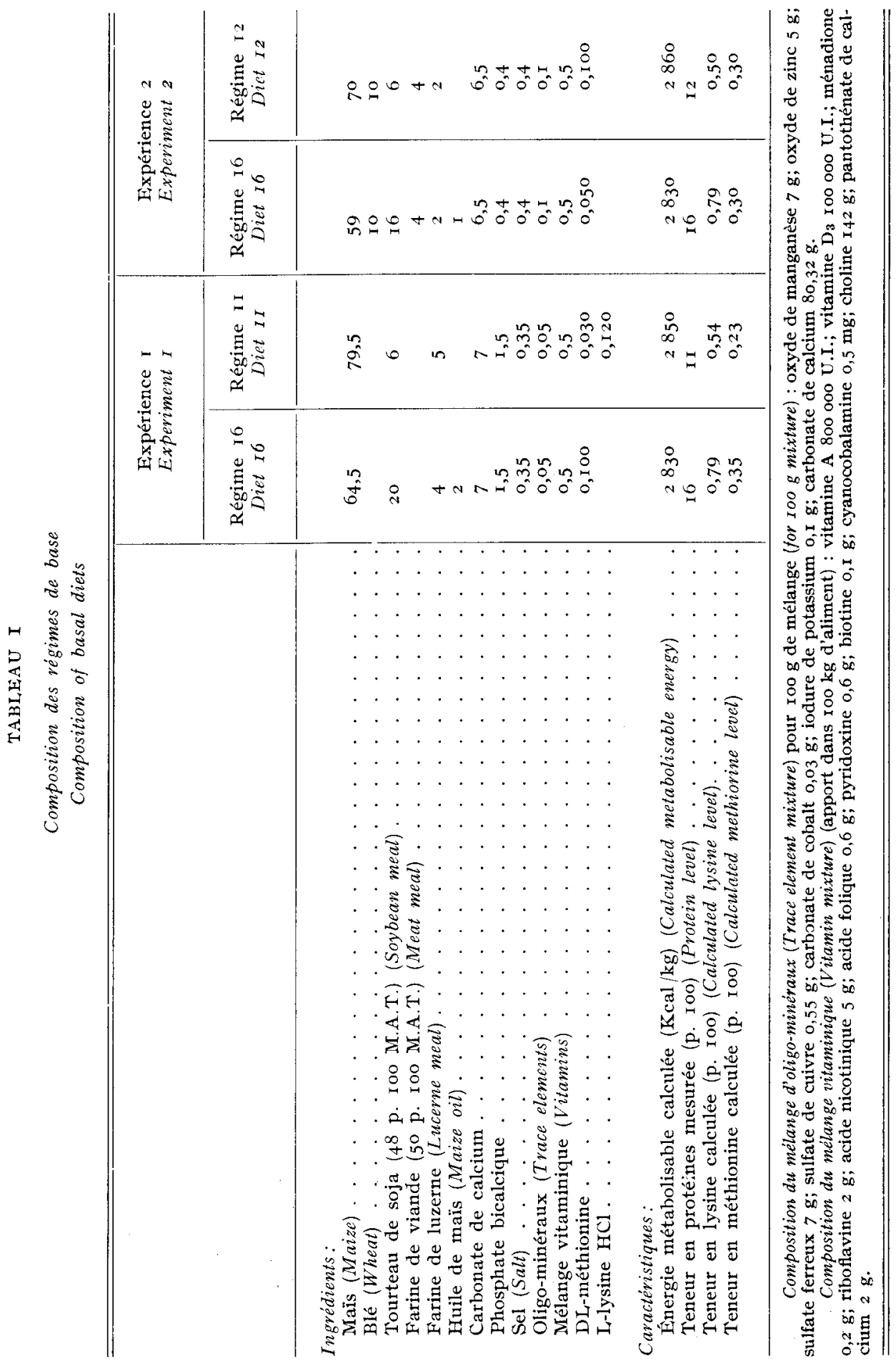




\section{Matériel et méthodes}

Deux expériences ont été entreprises, l'une en I975, l'autre en I978. Au cours de la première, on a comparé 6 régimes différant par leur teneur en protéines (II, I2, I3, I4, I5 et I6 p. IOo), les proportions de lysine et de méthionine étant maintenues constantes (respectivement 4,9 et 2 , I p. Ioo des matières azotées totales) par addition de lysine ou de méthionine libres. La composition des régimes de base est rapportée dans le tableau $\mathrm{r}$. Les régimes intermédiaires sont obtenus par mélange des deux régimes de base en proportions adéquates. Ces régimes ont été distribués quotidiennement à volonté ou en quantités limitées. Ce rationnement, calculé de façon à ne distribuer que $92 \mathrm{p}$. Ioo de la consommation des animaux nourris ad libitum, a été réalisé à la fin de $2^{\mathrm{e}}$ période de ponte, autrement dit après l'âge de 30 semaines. Chacun des 12 lots 6 régimes $\times 2$ modes de distribution) comportait I5 cages collectives $(48 \times 40 \mathrm{~cm})$, logeant chacune 3 poules.

La $2^{\mathrm{e}}$ expérience consiste en une distribution à volonté ou en quantité restreinte de 5 régimes différant par leur teneur en protéines (I2, I3, I4, I5 et r6 $\mathrm{p}$. roo). Ces régimes sont obtenus par mélange en proportions adéquates de 2 régimes de base dont la composition est rapportée aussi dans le tableau $\mathrm{I}$. Les régimes présentent tous la même teneur en méthionine $(0,30 \mathrm{p}$. Ioo). Ies conditions de rationnement sont exactement les mêmes que celles de la $I^{\text {re }}$ expérience. Chaque lot comprend i 8 cages collectives contenant chacune 3 poules.

Les pondetises sont de type Rhode Island Red (Warren I.S.A. France). A l'âge de $r 8$ semaines, elles sont réparties par groupe de 3 , la somme des poids étant semblable d'une cage à l'autre.

Les poules sont pesées à l'âge de 22 semaines (près du pic de ponte) et en fin d'expérience (62 semaines pour l'expérience $n^{0} \mathrm{I}, 70$ semaines pour l'expérience $\mathrm{n}^{0} 2$ ) après un jê̂ne total de 20 heures.

On contrôle le nombre d'œufs obtenus chaque jour de chaque cage. Le poids moyen est déterminé toutes les 4 semaines en pesant tous les œufs pondus pendant 4 jours successifs. Enfin, la consommation des lots nourris à volonté est mesurée par période de 4 semaines. L'aliment est distribué souts forme de farine. La teneur en protéines des régimes de base est ajustée à la valeur prévue (addition de tourteau de soja ou d'un mélange isoénergétique amidon + cellulose) après dosage (MacroKjeldahl) du régime maintenı dans la mélangeuse. De cette façon, les régimes présentent la même teneur en protéines durant toute l'expérience ( $\pm 0,05 \mathrm{p}$. I00). Keuls.

Les comparaisons entre moyennes sont effectuées par le test de Newman-

Les 2 expériences se sont déroulées dans un bâtiment chauffé dont la température ne descend pas en dessous de $18^{\circ} \mathrm{C}$. Le programme lumineux est du type classique; il assure I 6 heures de lumière par jour.

\section{Résultats}

Dans le tableau 2, nous récapitulons les résultats de $1^{\prime}$ expérience $\mathrm{n}^{\circ} \mathrm{I}$. Le nombre d'œufs pondus est influencé à la fois par la teneur du régime en protéines et par le rationnement; on ne met pas en évidence d'interaction significative. Peu élevé pour les teneurs faibles, ce critère de ponte n'est pas amélioré significativement lorsque le régime renferme plus de $15 \mathrm{p}$. Ioo de protéines. Le poids 


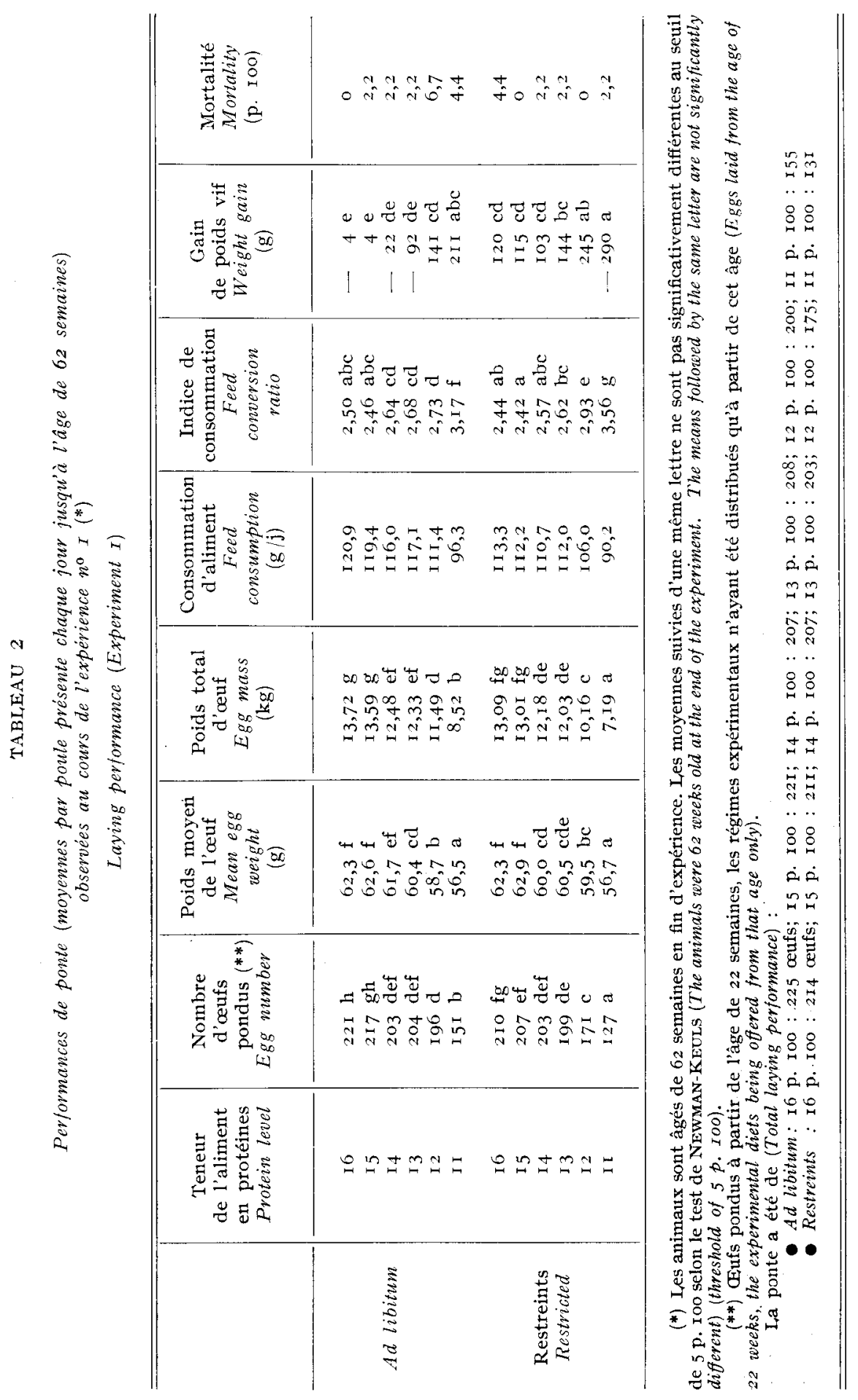


moyen de l'œuf est sous la seule dépendance de la composition du régime, le rationnement n'exerçant aucun effet. I, poids total d'œuf prođuit, combinaison des 2 critères précédents, est diminué aussi bien par la réduction des apports azotés que par le rationnement, sans que l'interaction statistique de ces 2 facteurs ne soit significative. L'ingestion alimentaire est évidemment différente selon que les animaux sont restreints ou non; mais on note aussi une réduction de la consommation si le régime renferme moins de I3 $\mathrm{p}$. Ioo de protéines. L'indice de consommation (kg d'aliment par $\mathrm{kg}$ d'œuf produit) n'est pas amélioré significativement par le rationnement, bien que les valeurs moyennes soient inférieures chez les poules restreintes recevant les 4 régimes les plus riches en protéines. Les régimes exercent, eux, des effets préjudiciables très significatifs lorsque la teneur en protéines descend en dessous de I $_{5} \mathrm{p}$. roo. Une interaction significative existe entre les 2 variables (apport azoté-rationnement); le rationnement des 2 régimes les plus pauvres détériore l'indice contrairement à ce qui se passe avec les régimes les plus riches. Enfin, la variation de poids vif mesurée à partir du pic de ponte est faible. Généralement, il s'agit d'une perte de poids d'autart plus forte chez les animaux nourris ad libitum que l'apport azoté est réduit.

Les résultats récapitulatifs de la $2^{\text {e }}$ expérience sont rassemblés dans 13 tableau 3. Comme dans l'expérience précédente, le nombre d'œufs pondus est sous la dépendance des régimes et du rationnement. Les poules recevant le régime à 12 p. roo de protéines n'ont pas été rationnées du fait de la faible consommation observée en alimentation ad libitum et de la médiocrité de leurs performancc; qui distingue nettement ce lot de tous les autres. Aucune interaction n'existe

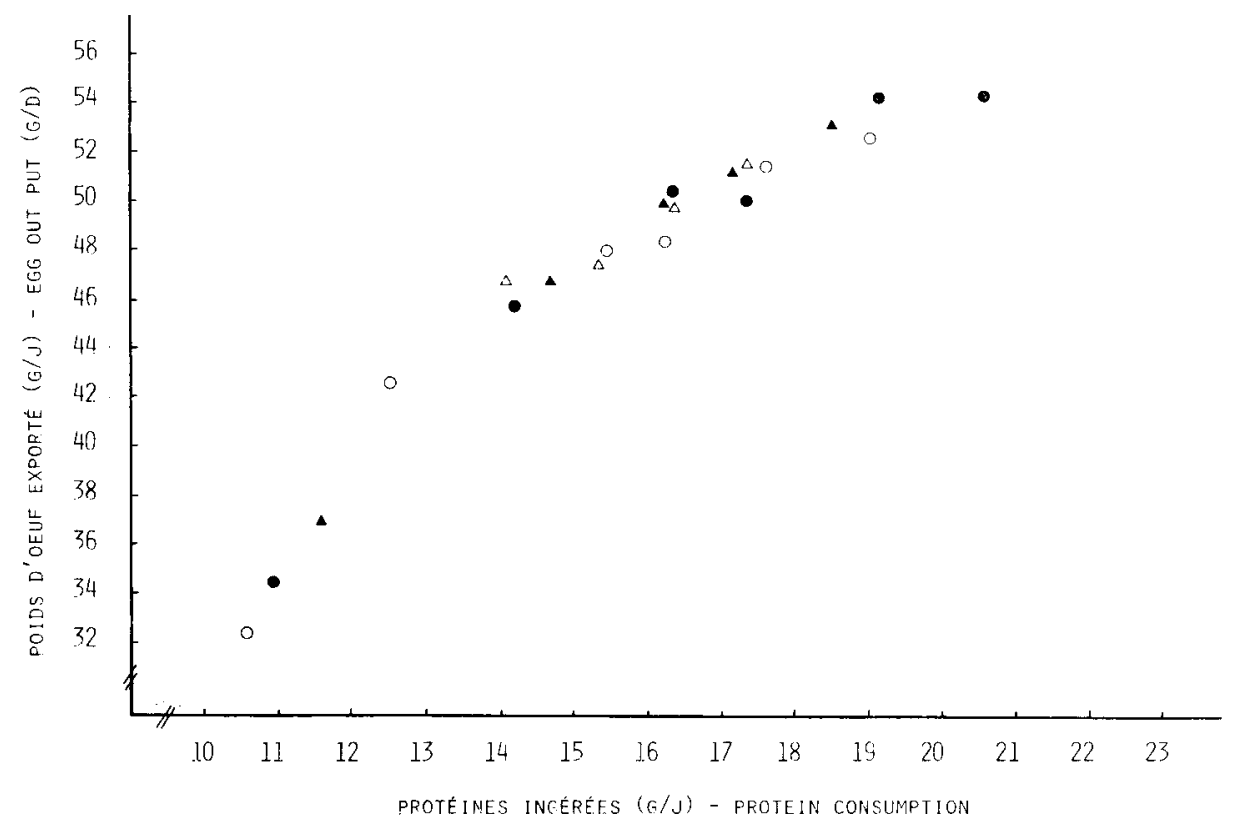

FIG. I. - Influence de la consommation quotidienne de protéines sur la production d'ceuts entre $3^{\circ}$ et ${ }_{4} 2$ semaines d'âge.

Infuence of daily protein consumption on egg output between 30 and 42 weeks of age.

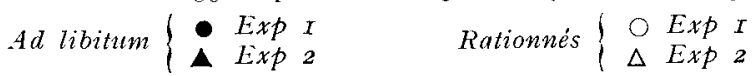




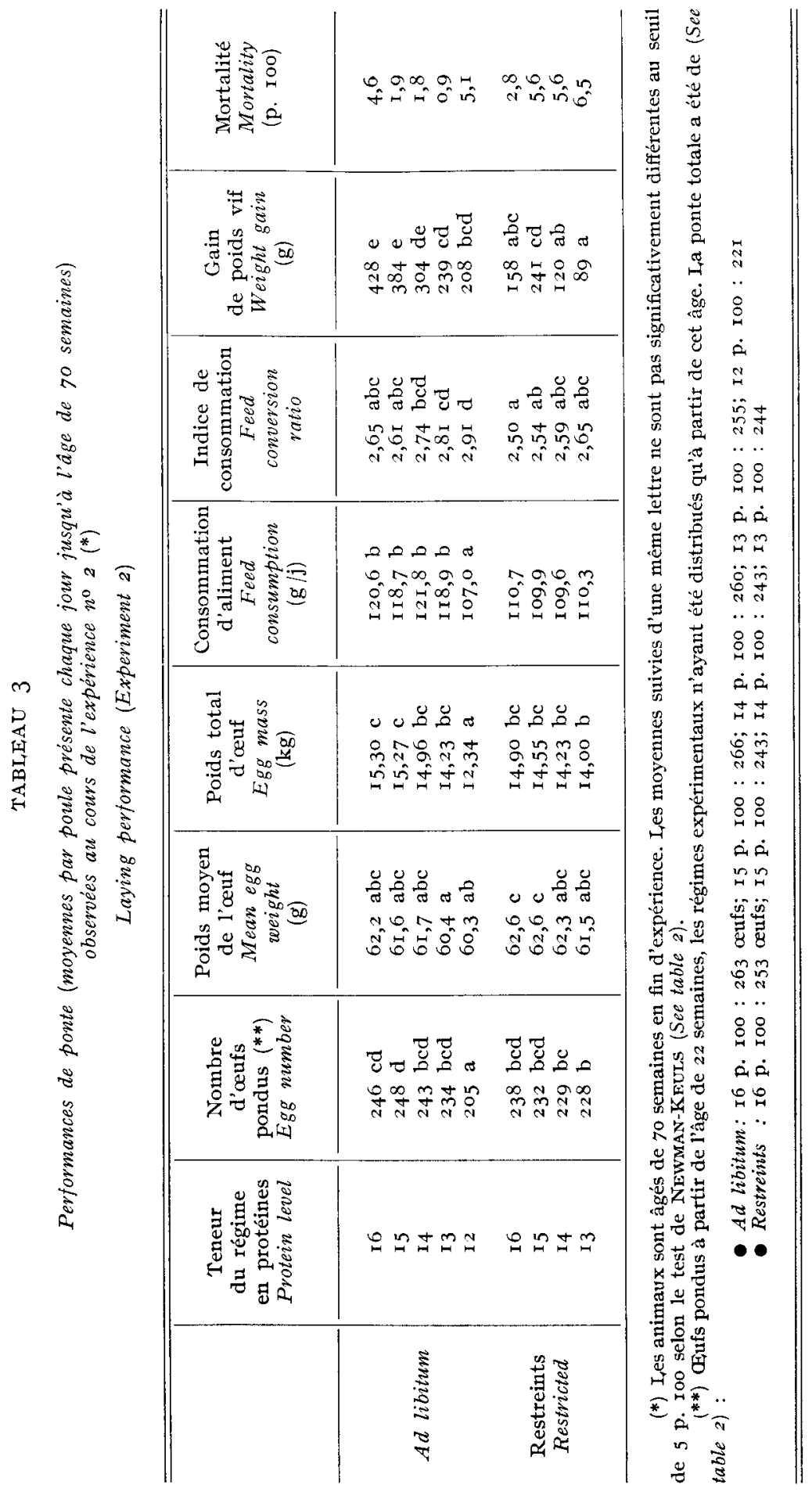


entre régimes et mode d'alimentation. Le poids moyen de l'œuf est modifié en sens opposé par la réduction des apports azotés et par la restriction alimentaire. L'influence favorable du rationnement sur le poids de l'œuf se retrouve au sein de chaque régime au cours de la plupart des périodes de ponte (résultats partiels non publiés). L'influence négative des apports azotés est un peu moins prononcée que dans l'expérience précédente. Le poids total d'œufs pondus reflète les modifications subies par les 2 critères de ponte précédents; les diminutions dues au rationnement sont atténuées, le déficit en nombre d'œufs étant compensé en partie par un poids moyen plus élevé. Dans cette $2^{\mathrm{e}}$ expérience, 1'indice de consommation est significativement amélioré par le rationnement quel que soit le régime distribué. Les régimes exercent aussi des effets significatifs, les apports azotés les plus faibles augmentant l'indice. Enfin, les gains de poids vif, nettement plus élevés que précédemment, sont réduits par le rationnement et par l'abaissement du taux protidique.

Nous illustrons aussi par la figure I les relations qui ont été observées entre la quantité d'œufs produite en moyenne par poule au cours des $\mathbf{2} 2$ semaines qui suivent le début du rationnement (de 30 à 42 semaines) et la quantité de protéines consommée, que les animaux soient rationnés ou non. On constate une remarquable superposition des 2 expériences. De plus, on note que les lots rationnés se situent sur la même courbe. Bien qu'on ne parvienne pas de façon très nette à un plateau, on peut tout de même remarquer qu'en alimentation ad libitum I9,I à $20,5 \mathrm{~g}$ de protéines assurent une production quotidienne identique et maximum de $54,5 \mathrm{~g}$.

Les taux de mortalité enregistrés lors de chaque expérience sont présentés dans les tableaux 2 et 3 sans qu'il soit possible de dégager des effets nets.

\section{Discussion}

Les résultats de ces 2 expériences peuvent être une source d'informations nouvelles en ce qui concerne, d'une part le rationnement et, d'autre part, le besoin en protéines de la poule pondeuse Warren.

\section{I. - Le rationnement de la pondeuse}

Comme nous l'avions déjà constaté précédemment (LECLERCQ, BoucharDEAU et BLUM, I975), il est clair que rationner les pondeuses Warren à 92 p. Ioo de la consommation spontanée entraîne une réduction systématique du nombre d'œufs. Au contraire, le rationnement n'altère pas le poids moyen de l'œuf, quel que soit le taux protidique. Nous observons même, dans la $2^{\mathrm{e}}$ expérience, une augmentation de ce poids moyen sous l'effet du rationnement, augmentation que nous ne parvenons pas à expliquer et qui n'est pas un artefact puisqu'on l'a retrouvée à tous les âges et avec tous les régimes. On sait que le rationnement tend à diminuer la production en affectant selon les cas, soit le poids, soit le nombre des oufs pondus (WELLS, r977). Cependant, une augmentation significative du poids de l'œuf n'avait pas encore été signalée. Elle est sans doute liée à la diminution de l'intensité de ponte qui raccourcit les séquences et peut ainsi réduire la proportion de petits œufs. Un rationnement alimentaire de la sévérité de celui 
appliqué ici améliore l'efficacité alimentaire, surtout dans 1a $2^{\mathrm{e}}$ expérience qui a duré plus longtemps. Cette amélioration n'apparaît qu'avec des régimes renfermant plus de 13 p. Ioo de protéines. En dessous de ce seuil, le rationnement aggrave les effets de la déficience azotée.

Les résultats de la figure I portant sur les I2 premières semaines de rationnement correspondent à une période où, la capacité de production des pondeuses étant la plus élevée, les exigences sont plus grandes (Shaptro, I968). De la figure I il ressort que l'ingéré énergétique n'est pas le facteur limitant dans ces 2 essais. C'est la quantité de protéines ingérées qui détermine les capacités de ponte. Les baisses de performances provoquées par le rationnement résultent de la réduction des apports protidiques. Ainsi, pendant cette période qui suit le pic de ponte, le rationnement exigerait l'emploi d'un régime riche en protéines. Cependant, il faut nuancer cette constatation en se souvenant qu'une période ne rend pas compte du déroulement de toute la ponte. Sur l'ensemble de la production (tab1. 2 et 3 ), le rationnement n'a pas une influence comparable à celle des apports protidiques réduits : le premier tend à augmenter le poids moyen de 1'œuf, les seconds le diminuent. En outre, seuls les taux protidiques très faibles ont une influence en fin de ponte, de sorte que le rationnement intervient dans les autres cas en améliorant l'indice de consommation, donc l'utilisation des nutriments énergétiques. Au total, le rationmement intervient un peu en limitant l'apport protidique en début de ponte et beaucoup sur l'ensemble de la période production en réduisant la consommation énergétique.

\section{2. - Le besoin en protéines}

Il ressort de la figure I que, pour sa période de performance maximum, la poule pondeuse de ce type (croisement Rhode Island) exige plus de I9 g de protéines par jour lorsque le régime est constitué d'un mélange de céréales (principalement le maïs), de tourteau de soja et de méthionine. L'équilibre différent des acides aminés au cours des 2 expériences ne paraît pas modifier le besoin en protéines; l'excès de méthionine an-delà de 0,30 p. roo (régime 16 de l'expérience I) ou la supplémentation en lysine des régimes pauvres en protéines (expérience I) ne modifie pas les liaisons entre production d'œufs et quantité de protéines ingérées.

Cependant, lorsqu'on prend en considération l'ensemble de la production, le besoin semble moins élevé : de l'ordre de $\mathrm{I} 8 \mathrm{~g}$ dans le $\mathrm{I}^{\mathrm{er}}$ essai puisqu'il peut être couvert avec I $5 \mathrm{p}$. Ioo de protéines, de $\mathrm{I} 6 \mathrm{~g} / \mathrm{j}$ dans la $2^{\mathrm{e}}$ expérience oì $\mathrm{I} 4 \mathrm{p}$. I 00 de protéines suffisent, sans doute parce que l'apport de méthionine est plus élevé.

Incontestablement, le besoin en protéines dépend à la fois du niveau de production et de l'équilibre des acides aminés. Utilisant les protéines d'œuf (en principe les mieux équilibrées), SHapiro (I968) avait constaté que l'apport azoté nécessaire pour assurer un bilan positif variait selon l'intensité de ponte; à 80 p. roo de production, il fallait I6 g de protéines par jour contre $\mathrm{I} 3 \mathrm{~g}$ à $67 \mathrm{p}$. Ioo. Ces résultats laisseraient supposer un besoin variable tout au long de la ponte. En fait, aucun avantage n'a été retiré de l'usage des taux protidiques variables (Mullar et Smith, I975). Dans les 2 essais que nous présentons, il semble que par compensation les poules sur régimes moyennement pourvus en protéines aient davantage pondu en fin de ponte. Dans l'expérience 2 faisant appel à des régines supplémentés en méthionine, la diminution du poids total d'œufs pondus n'atteint pas tout à fait le seuil de signification avec I3 p. roo de protéines. Cepen- 
dant, cet apport azoté n'assure pas un indice de consommation élevé. On retrouve un effet caractéristique des apports insuffisants d'acides aminés qui accroissent l'indice de consommation sans que le gain de poids vif augmente pendant la ponte (HAMILTON, I968).

En définitive, des apports très faibles de protéines ont pu être trouvés satisfaisants : II $\mathrm{g} / \mathrm{j}$ avec un mélange équilibré supplémenté en acides aminés (E, Boushy et MuirwiJk, I978). Cependant, de nombreux facteurs peuvent modifier les besoins. Les divergences observées dans la littérature sont liées au génotype des poules utilisées, à la qualité variable des aliments et des matières premières, au niveau de production ainsi qu'à la période et la durée des essais. Il est prudent de prévoir un apport un peu plus élevé que le besoin minimum, de 1'ordre de $\mathrm{I} 6 \mathrm{~g}$, avec les mélanges classiques à base de maïs et de tourteau de soja, simplement supplémentés en méthionine.

Accepté pour publication en février 1980 .

\section{Summary}

\section{Feed restriction of laying hens using different dietary protein levels}

During 2 experiments including 540 Rhode Island Red laying hens, diets containing different protein levels were offered ad libitum or restricted. The feed restriction did not change or even slightly increased the mean egg weight. It improved the feed conversion ratio, but always led to a reduction of the egg number whatever the protein level.

During the first months of restriction the effects observed were only connected with the level of protein intake; the requirement was then estimated to $20 \mathrm{~g} / \mathrm{day} / \mathrm{hen}$ (maize, soybean, methionine). However, for the whole laying period, the requirement of the hen did not exceed $\mathrm{r} 8 \mathrm{~g}$ and the variable protein deficiency cannot fully account for the results obtained. The effect of the feed restriction remains specific.

\section{Références bibliographiques}

Bougon M., Méver M., I972. Influence du rationnement en période de production sur les performances de trois variétés commerciales de pondeuses. Bull. Inf. Ploufragan, 12, i 54-160.

EL-Boushy A. R., Muinwijk I., 1978. Effect of varied protein levels with synthetic aminoacids on performance of layers and egg quality. Feedstufs, 50 (43), I 5-I 6.

HAMITON R. M. G., 1978. The effects of dietary protein level on productive performance and egg quality of four strains of White Leghorn hens. Poult. Sci., 57, I355.

I.ECLERCQ B., Bouchardeau A., BLUM J. C., 1975. Étude des effets du rationnement alimentaire de la poule sur ses performances de ponte et observations sur les effets d'une supplémentation par la Bacitracine. Ann. Zootech., 24, 603-6II.

MILI,AR R. I., SMTTH L. 'T., 1975. The effect of four different feeding programs of varying protein levels on performance of brown-egg type birds. Poult. Sci., 54, 964-968.

SHAPIRO R., 1968. Protein requirement, nitrogen retention and egg production of the laying hen. Fed. Proc., 27, 923-926.

WELLS R. G., I977. Improving the efficiency of food conversion in layers. Proc. Ist European Symp. Poult. Nutr., 8I-88 (published Danish branch of W.P.S.A.). 